\title{
ON MODELLING AND PRICING WEATHER DERIVATIVES DRIVEN BY NONLINEAR BROWNIAN MOTION
}

\author{
JAVED HUSSAIN*, PERVEZ ALI \\ Department of Mathematics, Sukkur IBA University, Sindh, Pakistan \\ *Corresponding author: javed.brohi@iba-suk.edu.pk
}

\begin{abstract}
In this paper, our focus is to derive the estimates satisfied by the risk-neutral prices of a class of weather derivatives, contingent upon temperature which satisfies $\mathbb{G}$-stochastic differential equation driven by nonlinear $\mathbb{G}$-Brownian motion.
\end{abstract}

\section{INTRODUCTION}

In this work, we aim to focus on the weather derivatives, HDD Call, and CDD call, where the underlying temperature is driven by a version of nonlinear Brownian motion, known as $\mathbb{G}$ - Brownian motion. Robust finance is one of the emerging areas of modern finance, where the focus is on developing the risk management models, where the underlying asset is driven by uncertain volatility. Classically, in the most risk management model/ financial asset pricing theory, the volatility is either assumed to be constant, deterministic or in case it is taken stochastic, it is driven by linear noise such as the Wiener process. In all of these cases, models suffer from reversal disadvantages such as mispricing of financial assets. One very interesting proposal was given by Levy in $[3,1995]$, to take the volatility to be uncertain i.e. lie in a closed interval, this study was a good start but suffered from the problem of risk management tools such as options were not dynamically priced. The solution to this problem came from Peng in $[24,2007]$, where he introduced the motion of the probability space with independent nonlinear expectation known as $\mathbb{G}$-Expectation. This

Received September $6^{\text {th }}, 2020$; accepted November $3^{\text {rd }}, 2020$; published November $24^{\text {th }}, 2020$.

2010 Mathematics Subject Classification. 60J65, 60J70.

Key words and phrases. financial derivatives; weather contracts; nonlinear Brownian motion.

(C)2021 Authors retain the copyrights of their papers, and all open access articles are distributed under the terms of the Creative Commons Attribution License. 
was the point of inception of $\mathbb{G}$-Stochastic Calculus and $\mathbb{G}$-measure-theoretic probability. In $[24,2007]$ and [25, 2008], Peng introduced this nonlinear version of probability and stochastics, more precisely Peng introduced $\mathbb{G}$-Conditional expectations, $\mathbb{G}$-normal distributions which can be treated as the solution of fully nonlinear $\mathbb{G}$-Heat equation. Peng also introduced the notion of $\mathbb{G}$-Brownian motion, $\mathbb{G}$-Stochastic integrals and their function spaces, $\mathbb{G}$-Martingale, $\mathbb{G}$-Sub \& Super Martingales, $\mathbb{G}$-Ito process, $\mathbb{G}$-stochastic differential equations i.e. SDE driven by $\mathbb{G}$-Brownian motion and multi-dimensional $\mathbb{G}$-Ito formulas. Zhao in [33, 2016] numerically simulated $\mathbb{G}$-normal distribution and $\mathbb{G}$-Brownian motion. In [27, 2019], Peng introduced the Feynman Kac formula which is pivotal in solving nonlinear probabilistic motions in $\mathbb{G}$-framework. Using all these tools in $\mathbb{G}$-framework, $\mathrm{Xu}$ in $[31,2010]$ and $[32,2011]$ studied the EU call option and Girsanov theorem in $\mathbb{G}$-framework. Recently Julian in $[15,2020]$ has developed the theory of interest rate derivative in $\mathbb{G}$-framework.

The weather puts impacts all kinds of business activities directly or indirectly, so naturally, it is significant to make good predictions about the weather. Production, transport, and preservation of agriculture crops; energy production, distribution (cf. [1, 2002]) and consumption; Chain of supermarkets, tourism, and leisure industries are directly influenced by the weather. Indeed, the key factors involved in weather that must be taken care of, include a variation of temperature, wind, rainfall, humidity level, snowfall, etc. In particular, we will focus on modeling the dynamics of temperature. The weather derivatives are becoming more and more popular due to several reasons. For instance, in the energy market, the energy producers are facing the challenges, firstly, as the energy sector is getting less and less regulated, and secondly since there is a positive correlation between the weather and the demand for energy (cf. [29, 2017]), hence, the prices of the energy is not the hands of energy produces. Indeed this creates competition in the energy market, among the producers, and therefore the energy companies are interested to hedge their risk by entering into financial contracts based in the weather. The weather derivative market is not very liquid, it looks like many companies have not yet define hedging policies or even figured out themselves to weather risk. If actors outside the energy sector take interest in the weather derivatives market there will be exponential growth potential. There are some barriers that must be removed if the market is to grow. for example, the quality and cost of weather data. Companies which want to analyze their performance against historical weather data, they should often buy information from the national meteorological offices and that is quite expensive. And the main issue is whether the gained information about the weather is good to rely on or not. The weather derivatives are a relatively new way to hedge the risk associated with weather, they were first introduced by Marginson in [20, 2000]. This gives rise to interest in carefully studying the seasonal weather events El Niño event (cf. Trenberth [30, 1997]), and make a good prediction about the weather so that seasonal risks can be hedged. After that, the weather derivative market expanded rapidly and contracts have been traded over-the-counter('OTC') as individually negotiated contracts, the primary user 
of weather derivatives were the energy sectors. Now the first market namely, Chicago Mercantile Exchange ('CME') started an electronic marketplace for weather derivatives in September 1999. The founder of CME is Aquila Energy, Koch Energy Trading, Southern Energy, Enron, and Castle bridge weather markets, All were active in the OTC market for weather derivatives. For more on weather derivatives we refer to recent works [18, 2011] [22, 2013], [4, 2013], [23, 2013], [21, 2019] and [28, 2020].

We now give a brief organization of the paper. Section 2 comprises a very brief introduction to $\mathbb{G}-$ Stochastic Calculus. Section 3 gives the reader a detailed account of the weather derivatives. Section 4 comprises of our key results or estimates satisfied by the risk-neutral prices of weather derivatives (HDD call and CDD Call), where the underlying non-trade able asset temperature is driven by $\mathbb{G}$-Brownian motion.

\section{Preliminaries}

2.1. Sublinear Expectation. Linear expectation $\mathbb{E}$ satisfies the following relations for random variables $\mathrm{X}$ and $\mathrm{Y}$;

$$
\mathbb{E}[a X]=a \mathbb{E}[X], \quad \forall \quad a \in \mathbb{R}, \quad \text { and } \quad \mathbb{E}[X+Y]=\mathbb{E}[X]+\mathbb{E}[Y]
$$

A sub-linear expectation $\widehat{\mathbb{E}}^{\mathbb{G}}$ satisfies the following weaker condition;

$$
\widehat{\mathbb{E}}^{\mathbb{G}}[a X]=a \widehat{\mathbb{E}}^{\mathbb{G}}[X] \quad \text { for } \quad a>0, \quad \widehat{\mathbb{E}}^{\mathbb{G}}[X+Y] \leq \widehat{\mathbb{E}}^{\mathbb{G}}[X]+\widehat{\mathbb{E}}^{\mathbb{G}}[Y] .
$$

The sublinear expectation $\widehat{\mathbb{E}}^{\mathbb{G}}$ follows the monotonicity property same as a linear expectation: if $X \geq Y$ then $\widehat{\mathbb{E}}^{\mathbb{G}}[X] \geq \widehat{\mathbb{E}}^{\mathbb{G}}[Y]$. The sublinear expectation is very important in volatility uncertainty, through sublinear expectation the people are doing work in super-hedging, super-pricing (cf. [3, 1995] and [19, 1995]) and measures of risk in finance which caused great attention in finance since the fundamental work of $[2,1999]$. In Sublinear expectation(non-linear expectation) space, One can introduce the distributions, of random variables, like distribution of a single variable, joint distribution, marginal distributions, etc. One still can show the independence and identically distributed random variables but in the sub-linear expectation if $X$ is independent to $Y$ it does not directly imply that $Y$ is independent to $X$. We can still prove some important theorems in sublinear expectation theory, The law of large numbers, and the central limit theorem, see $[24,2007]$. One can see the $\mathbb{G}$-expectation in $[26,2010]$ and $\mathbb{G}$-martingales in $[17,2008]$, also the Girsanov theorem under $\mathbb{G}$-framework in $[32,2011]$ is now available. $\mathrm{G}$ - Brownian motion has a very rich and interesting new structure that generalizes the classical structure without triviality. We can determine the related stochastic calculus, particularly the G - Itô integrals (see [16, 1987]).

A short introduction of sublinear expectation and some definitions are the part of this section.

Definition 2.1. (Sublinear Expectation [27, 2019]) Let $\chi$ be the linear subspace of real valued and bounded functions on $\Omega$ and a functional $\widehat{\mathbb{E}}^{\mathbb{G}}[$.$] on \chi$ satisfying the following properties is called sublinear expectation. 
1. Monotonicity:

$$
X \geq Y \Longrightarrow \widehat{\mathbb{E}}^{\mathbb{G}}[X] \geq \widehat{\mathbb{E}}^{\mathbb{G}}[Y]
$$

2. Constant preserving:

$$
\widehat{\mathbb{E}}^{\mathbb{G}}[c]=c, \quad \forall c \in \mathbb{R} .
$$

3. Sub-additivity: For every $X, Y \in \chi$

$$
\widehat{\mathbb{E}}^{\mathbb{G}}[X+Y] \leq \widehat{\mathbb{E}}^{\mathbb{G}}[X]+\widehat{\mathbb{E}}^{\mathbb{G}}[Y]
$$

4. Positive homogeneity:

$$
\widehat{\mathbb{E}}^{\mathbb{G}}[\lambda X]=\lambda \widehat{\mathbb{E}}^{\mathbb{G}}[X], \quad \forall \lambda \geq 0 .
$$

From the above properties(2.1) and(2.1) one can also show the convexity

$$
\widehat{\mathbb{E}}^{\mathbb{G}}[\alpha X+(1-\alpha) Y] \leq \alpha \widehat{\mathbb{E}}^{\mathbb{G}}[X]+(1-\alpha) \widehat{\mathbb{E}}^{\mathbb{G}}[Y], \quad \forall \alpha \in[0,1]
$$

Definition 2.2. [27, 2019] Let $\widehat{\mathbb{E}}^{\mathbb{G}, 1}$ and $\widehat{\mathbb{E}}^{\mathbb{G}, 2}$ be sublinear expectations if,

$$
\widehat{\mathbb{E}}^{\mathbb{G}, 1}[X]-\widehat{\mathbb{E}}^{\mathbb{G}, 1}[Y] \leq \widehat{\mathbb{E}}^{\mathbb{G}, 2}[X-Y], \quad \forall X, Y \in \chi
$$

The $\widehat{\mathbb{E}}^{\mathbb{G}, 2}$ is dominated by $\widehat{\mathbb{E}}^{\mathbb{G}, 2}$. From (2.1) of Definition 2.1 a nonlinear expectation is dominated by itself and the strongest nonlinear expectation on $\chi$ is

$$
\widehat{\mathbb{E}}^{\mathbb{G}, \infty}[X]:=\sup _{\omega \in \Omega} X(\omega)
$$

every sub linear expectation is dominated by the strongest sublinear expectation.

We will denote $\mathcal{P}_{f}$ set of of all finitely additive probability measures on $(\omega, \mathcal{F})$.

Definition 2.3. (Distribution in linear expectation) [27, 2019] Let $P \in \mathcal{P}_{f}$ and let $X: \Omega \mapsto \mathbb{R}$ be a $\mathcal{F}$ measurable function such that $|X(\omega)|<\infty$ for every $\omega$. The distribution of random variable $X$ on $(\Omega, \mathcal{F}, \mathbb{P})$ is defined as,

$$
\mathbb{F}_{X}[\varphi]=\mathbb{E}_{P}[\varphi(X)]: \varphi \in \mathbb{L}^{\infty}(\mathbb{R}, \mathcal{B}(\mathbb{R})) \mapsto \mathbb{R}
$$

Here equation (2.3) can be written as

$$
F_{X}[\varphi]=\int_{\mathbb{R}} \varphi(x) F_{X}(d x)
$$


Definition 2.4. [27, 2019] The distributions under nonlinear expectations, is defined as, let the random variable $X=\left(X_{1}, X_{2}, \ldots, X_{3}\right)$ be a n-dimensional random vector on a nonlinear expectation space $\left(\Omega_{1}, \mathscr{H}_{1}, \widehat{\mathbb{E}}^{\mathbb{G}}\right)$

$$
\widehat{\mathbb{F}}_{X}[\varphi]:=\widehat{\mathbb{E}}^{\mathbb{G}}[\varphi(X)]: \varphi \in C_{l . L i p}\left(\mathbb{R}^{n}\right) \mapsto(-\infty, \infty)
$$

where $C_{\text {l.Lip }}\left(\mathbb{R}^{n}\right)$ is the space of Lipschitz continuous functions. the following triple $\left(\mathbb{R}^{n}, C_{\text {l.Lip }}\left(\mathbb{R}^{n}\right), \widehat{\mathbb{F}}_{X}[\cdot]\right)$ is called sublinear expectation space. $\widehat{\mathbb{F}}_{X}$ is the distribution of $X$.

Definition 2.5. (Mean and Variance -Uncertainty) [27, 2019] In general, under sublinear expectation mean and variance are uncertain, in robust statistics $\widehat{\mathbb{E}}^{\mathbb{G}}[X] \neq-\widehat{\mathbb{E}}^{\mathbb{G}}[-X]$ similarly $\widehat{\mathbb{E}}^{\mathbb{G}}\left[X^{2}\right] \neq-\widehat{\mathbb{E}}^{\mathbb{G}}\left[-X^{2}\right]$, so now these are four different parameters

$$
\bar{\mu}:=\widehat{\mathbb{E}}^{\mathbb{G}}[X], \quad \widehat{\mu}:=-\widehat{\mathbb{E}}^{\mathbb{G}}[-X], \quad \bar{\sigma}^{2}:=\widehat{\mathbb{E}}^{\mathbb{G}}\left[X^{2}\right], \quad \widehat{\sigma}^{2}:=-\widehat{\mathbb{E}}^{\mathbb{G}}\left[-X^{2}\right] .
$$

The purpose of this study is to discuss only variance uncertainty if someone is interested to study the mean uncertainty he/she may (see [5, 2017] and [6, 2002]). So it is assumed that

$$
\widehat{\mathbb{E}}^{\mathbb{G}}[X]=-\widehat{\mathbb{E}}^{\mathbb{G}}[-X] .
$$

Proposition 2.1. [27, 2019] Let $X, Y \in \mathscr{H}$ and $\widehat{\mathbb{E}}^{\mathbb{G}}[Y]=-\widehat{\mathbb{E}}^{\mathbb{G}}[-Y]$ or $Y$ has not mean uncertain. Then

$$
\widehat{\mathbb{E}}^{\mathbb{G}}[X+Y]=\widehat{\mathbb{E}}^{\mathbb{G}}[X]+\widehat{\mathbb{E}}^{\mathbb{G}}[Y] .
$$

Definition 2.6. (Independence in sublinear expectation) [27, 2019] Notion of independence in sublinear expectation space $\left(\mathbb{R}^{n}, C_{\text {l.Lip }}\left(\mathbb{R}^{n}\right), \widehat{\mathbb{E}}^{\mathbb{G}}\right)$ is same as linear expectation space. Let a random vector $Y=$ $\left(Y_{1}, \ldots, Y_{n}\right), Y_{i} \in \mathscr{H}$ is said to be independent to random vector $X=\left(X_{1}, \ldots, X_{m}\right), X_{i} \in \mathscr{H}$ under $\widehat{\mathbb{E}}^{\mathbb{G}}[\cdot]$ if for every function $\varphi \in C_{l . L i p}\left(\mathbb{R}^{m} \times \mathbb{R}^{n}\right)$, the independence can be expressed as,

$$
\widehat{\mathbb{E}}^{\mathbb{G}}[\varphi(X, Y)]=\widehat{\mathbb{E}}^{\mathbb{G}}\left[\widehat{\mathbb{E}}^{\mathbb{G}}[\varphi(x, Y)]_{x=X}\right]
$$

2.2. G-normal distribution. In this section, some definitions has been discussed related to normal distribution under sublinear expectation as defined in [24, 2007].

Definition 2.7. $\mathbb{G}$-normal distribution: [27, 2019] $A$ random variable $X \in \mathscr{H}$ in sublinear expectation space $\left(\Omega, \mathscr{H}, \widehat{\mathbb{E}}^{\mathbb{G}}\right)$ with $\bar{\sigma}^{2}=\widehat{\mathbb{E}}^{\mathbb{G}}\left[X^{2}\right], \quad \widehat{\sigma}^{2}=-\widehat{\mathbb{E}}^{\mathbb{G}}\left[-X^{2}\right]>0$ is called $\mathcal{N}\left(0 ;\left[\widehat{\sigma}^{2}, \bar{\sigma}^{2}\right]\right)$-distributed, if for every $Y \in \mathscr{H}$ independent to $X, Y \sim X$ and $a X+b Y \sim \sqrt{a^{2}+b^{2}} X, \quad \forall a, b \geq 0$.

From above definition this is clear that $\widehat{\mathbb{E}}^{\mathbb{G}}[X]=-\widehat{\mathbb{E}}^{\mathbb{G}}[-X]=0$ hence random variable $X$ has no mean uncertainty.

The $\mathbb{G}$-normal distribution $\mathcal{N}\left(0 ;\left[\widehat{\sigma}^{2}, \bar{\sigma}^{2}\right]\right)$ is generated by the parabolic PDE defined for $[0, \infty) \times \mathbb{R}$ :

$$
\partial_{t} u-G\left(\partial_{x x}^{2} u\right)=0
$$


with Cauchy condition $\left.u\right|_{t=0}=\varphi$ where $G$,

$$
G(\alpha):=\frac{1}{2} \widehat{\mathbb{E}}^{\mathbb{G}}\left[X^{2} \alpha\right]=\frac{1}{2}\left(\bar{\sigma}^{2} \alpha^{+}-\widehat{\sigma}^{2} \alpha^{-}\right), \alpha \in \mathbb{R}
$$

is called the generating function.

Where $\alpha^{+}:=\max 0, \alpha$ and $\alpha^{-}:=\max 0,-\alpha$, the equation (2.6) is called generating heat equation.

The solution of equation (2.6) is defined as

$$
u(t, x):=\widehat{\mathbb{E}}^{\mathbb{G}}[\varphi(x+\sqrt{t} X)], \quad(t, x) \in[0, \infty) \times \mathbb{R} .
$$

The $\mathbb{G}$-heat equations can be also written in the form,

$$
\partial_{t} u-\frac{1}{2}\left(\bar{\sigma}^{2}\left(\partial_{x x}^{2} u\right)^{+}-\widehat{\sigma}^{2}\left(\partial_{x x}^{2} u\right)^{-}\right)=0 .
$$

2.3. $\mathbb{G}-B$ rownian Motion. $[27,2019] \mathbb{G}$-Brownian motion with respect to the $\mathbb{G}$-normal distribution in a sublinear expectation space is defined as,

Definition 2.8. ( $\mathbb{G}$-Brownian Motion) A process $\left(B_{t}^{\mathbb{G}}(\omega)\right)_{t \geq 0}$ is called $\mathbb{G}$-Brownian motion, if for $n \in \mathbb{N}$, $t_{1}<t_{2}<, \ldots,<t_{n}$ and $B_{t_{1}}^{\mathbb{G}}, B_{t_{2}}^{\mathbb{G}}, \ldots, B_{t_{n}}^{\mathbb{G}} \in \mathscr{H}$ it satisfies the following conditions,

- $B_{0}^{\mathbb{G}}(\omega)=0$

- For every $t, s \geq 0, B_{t+s}^{\mathbb{G}}-B_{t}^{\mathbb{G}}$ is $\mathcal{N}\left(0 ;\left[\widehat{\sigma}^{2} s, \bar{\sigma}^{2} s\right]\right)$-distributed and independent to $\left(B_{t_{1}}^{\mathbb{G}}, B_{t_{2}}^{\mathbb{G}}, \ldots, B_{t_{n}}^{\mathbb{G}}\right)$

$G$ is the same as it was defined in section 2.7. It will be used, for the sake of simplicity, without loss of generality, in this work, let $\bar{\sigma}=1$ and $\widehat{\sigma} \leq 1$, by this assumption now

$$
G(\alpha):=\frac{1}{2}\left(\alpha^{+}-\widehat{\sigma}^{2} \alpha^{-}\right), \quad \alpha \in \mathbb{R}
$$

Now $B_{t}^{\mathbb{G}} \sim \mathcal{N}\left(0 ;\left[\widehat{\sigma}^{2} s, s\right]\right)$. The existence of $\mathbb{G}$-Brownian motion has been proven in [24, 2007].

Definition 2.9. (G-Expectation) [27, 2019] Sublinear expectation is also called $\mathbb{G}$-expectation. The canonical process $\left(B_{t}^{\mathbb{G}}\right)_{t \geq 0}$ in the sublinear expectation space $\left(\Omega, \mathscr{H}, \widehat{\mathbb{E}}^{\mathbb{G}}\right)$ is $\mathbb{G}$-Brownian process.

There are some properties conditional $\mathbb{G}$-expectations which can be helpful in our study, let for any $X, Y \in \mathscr{H}^{0}$ here $\mathscr{H}^{0}$ is the used for $L_{i p}^{0}(\mathcal{F})$,

(1) $X \geq Y \Longrightarrow \widehat{\mathbb{E}}^{\mathbb{G}}\left[X \mid \mathscr{H}_{t}\right] \geq \widehat{\mathbb{E}}^{\mathbb{G}}\left[Y \mid \mathscr{H}_{t}\right]$

(2) $\widehat{\mathbb{E}}^{\mathbb{G}}\left[\eta \mid \mathscr{H}_{t}\right]=\eta, \quad$ for every $t \in[0, \infty)$ and $\eta \in \mathscr{H}_{t}^{0}$,

(3) For every $X, Y \in \chi, \widehat{\mathbb{E}}^{\mathbb{G}}\left[X \mid \mathscr{H}_{t}\right]-\widehat{\mathbb{E}}^{\mathbb{G}}\left[Y \mid \mathscr{H}_{t}\right] \leq \widehat{\mathbb{E}}^{\mathbb{G}}\left[X-Y \mid \mathscr{H}_{t}\right]$.

(4) $\widehat{\mathbb{E}}^{\mathbb{G}}[\eta X]=\eta^{+} \widehat{\mathbb{E}}^{\mathbb{G}}\left[X \mid \mathscr{H}_{t}\right]+\eta^{-\widehat{\mathbb{E}}^{\mathbb{G}}}\left[-X \mid \mathscr{H}_{t}\right], \quad$ for every $\eta \in \mathscr{H}_{t}^{0}$.

Sublinear expectation theory also have the Tower property:

$$
\widehat{\mathbb{E}}^{\mathbb{G}}\left[\widehat{\mathbb{E}}^{\mathbb{G}}\left[X \mid \mathscr{H}_{t}\right] \mid \mathscr{H}_{s}\right]=\widehat{\mathbb{E}}^{\mathbb{G}}\left[X \mid \mathscr{H}_{t \wedge s}\right]
$$


and

$$
\widehat{\mathbb{E}}^{\mathbb{G}}\left[X+\eta \mid \mathscr{H}_{t}\right]=\widehat{\mathbb{E}}^{\mathbb{G}}[X]+\eta, \quad \text { for every } t \in[0, \infty) \text { and } \eta \in \mathscr{H}_{t}^{0}
$$

The Proposition 2.1 can be defined similarly in the conditional $\mathbb{G}$-expectation. Some moments of $\mathbb{G}-$ Brownian motion's increments are, $\widehat{\mathbb{E}}^{\mathbb{G}}\left[B_{t}^{\mathbb{G}}-B_{s}^{\mathbb{G}} \mid \mathscr{H}_{s}\right]=0$, for every $s<t, n^{\text {th }}$ moment of increments is

$$
\widehat{\mathbb{E}}^{\mathbb{G}}\left[\left|B_{t}^{\mathbb{G}}-B_{s}^{\mathbb{G}}\right|^{n} \mid \mathscr{H}_{s}\right]=\frac{1}{\sqrt{2 \pi(t-s)}} \int_{-\infty}^{\infty}|x|^{n} \exp -\frac{x^{2}}{2(t-s)} d y
$$

But,

$$
\widehat{\mathbb{E}}^{\mathbb{G}}\left[-\left|B_{t}^{\mathbb{G}}-B_{s}^{\mathbb{G}}\right|^{n} \mid \mathscr{H}_{s}\right]=-\widehat{\sigma}^{n} \widehat{\mathbb{E}}^{\mathbb{G}}\left[\left|B_{t}^{\mathbb{G}}-B_{s}^{\mathbb{G}}\right|^{n} \mid \mathscr{H}_{s}\right]
$$

Now these are the formulas for $n^{\text {th }}$ moment, so one can easily calculate the moment which is needed, just like the classical case.

Definition 2.10. (G-Martingale) [27, 2019] An $\left(M_{t}\right)_{t \geq 0}$ process is said to be $\mathbb{G}$-martingale if for every $t \in[0, \infty), M_{t} \in \mathscr{H}^{0}$ and for every $t \in[0, t]$,

$$
\widehat{\mathbb{E}}^{\mathbb{G}}\left[M_{t} \mid \mathscr{H}_{s}\right]=M_{s}
$$

Similarly, $\mathbb{G}$-submartingale and $\mathbb{G}$-super-martingale are defined as, $\widehat{\mathbb{E}}^{\mathbb{G}}\left[M_{t} \mid \mathscr{H}_{s}\right] \geq M_{s}$ and $\widehat{\mathbb{E}}^{\mathbb{G}}\left[M_{t} \mid \mathscr{H}_{s}\right] \leq M_{s}$ respectively.

Example 2.1. Processes $\left(B_{t}^{\mathbb{G}}\right)_{t \geq 0}$ and $\left(-B_{t}^{\mathbb{G}}\right)_{t \geq 0}$ are $\mathbb{G}$-Martingales and $\left(\left(B_{t}^{\mathbb{G}}\right)^{2}\right)_{t \geq 0}$ is $\mathbb{G}$-submartingale.

In classical Brownian motion the quadratic variation of Brownian motion is a deterministic function but, in $\mathbb{G}$-Brownian motion the quadratic variation is itself a process. The definition of quadratic variation (cf. $[27,2019])$ is,

$$
\left\langle B^{\mathbb{G}}\right\rangle_{t}=B_{t}^{\mathbb{G}^{2}}-2 \int_{0}^{t} B_{s}^{\mathbb{G}} d B_{s}^{\mathbb{G}}
$$

One can easily verify that,

$$
\begin{aligned}
\widehat{\mathbb{E}}^{\mathbb{G}}\left[\left\langle B^{\mathbb{G}}\right\rangle_{t}-\left\langle B^{\mathbb{G}}\right\rangle_{s} \mid \mathscr{H}_{t}\right] & =t-s, \\
\widehat{\mathbb{E}}^{\mathbb{G}}\left[-\left(\left\langle B^{\mathbb{G}}\right\rangle_{t}-\left\langle B^{\mathbb{G}}\right\rangle_{s}\right) \mid \mathscr{H}_{t}\right] & =-\widehat{\sigma}^{2}(t-s) .
\end{aligned}
$$

Following some lemmas are being written without proofs, for proofs (see [24, 2007]) 
Lemma 2.1. [27, 2019] a) For every $s \geq 0,\left(\left\langle B^{\mathbb{G}}\right\rangle_{s+t}-\left\langle B^{\mathbb{G}}\right\rangle_{s}\right)_{t \geq 0}$ is independent of $\mathcal{F}_{s}$. This is the quadratic variation process of the Brownian motion $B_{t}^{\mathbb{G}^{s}}=B_{s+t}^{\mathbb{G}}-B_{s}^{\mathbb{G}}, t \geq 0$, i.e., $\left\langle B^{\mathbb{G}}\right\rangle_{s+t}-\left\langle B^{\mathbb{G}}\right\rangle_{s}=\left\langle B^{\mathbb{G}^{s}}\right\rangle_{t}$, Moreover, $\widehat{\mathbb{E}}^{\mathbb{G}}\left[\left\langle B^{\mathbb{G}}\right\rangle_{t}^{2}\right] \leq 10 t^{2}$.

b) For square integrable process $\left(\eta_{t}\right)_{t \geq 0}$ in the sense $\int_{0}^{T} \widehat{\mathbb{E}}^{\mathbb{G}}\left[\left|\eta_{t}\right|^{2}\right] d t$, then

$$
\begin{aligned}
\widehat{\mathbb{E}}^{\mathbb{G}}\left[\int_{0}^{T} \eta(s) d B_{s}\right] & =0, \\
\widehat{\mathbb{E}}^{\mathbb{G}}\left[\left(\int_{0}^{T} \eta(s) d B_{s}\right)^{2}\right] & \leq \int_{0}^{T} \widehat{\mathbb{E}}^{\mathbb{G}}\left[(\eta(t))^{2}\right] d t, \\
\widehat{\mathbb{E}}^{\mathbb{G}}\left[\int_{s}^{t} \eta_{u} d\left\langle B^{\mathbb{G}}\right\rangle_{u} \mid \mathcal{F}_{s}\right] & \leq \int_{s}^{t}\left|\eta_{u}\right| d u .
\end{aligned}
$$

The distribution of quadratic variation contains mean and variance uncertainty, see equation (2.16) and above lemmas.

2.4. Ito formula for $\mathbb{G}$-Brownian motion. Like classical Brownian motion, Itô’'s formula and integral can be defined under $\mathbb{G}$-Brownian motion, that is,

Theorem 2.1. (Ito's formula for $\mathbb{G}$-Brownian motion) [27, 2019] Let the $\mathbb{G}$-Itô process of $X$ is of form,

$$
\left.X_{t}=X_{s}+\alpha_{t} d t+\eta_{t} d\left\langle B^{\mathbb{G}}\right\rangle_{s}\right)_{t}+\beta_{t} d B_{t}^{\mathbb{G}}
$$

Then the $\mathbb{G}$-Itô formula of $\Phi\left(X_{t}\right)$ is given as,

$$
\begin{aligned}
\Phi\left(X_{t}\right)=\Phi\left(X_{s}\right)+\int_{s}^{t} \alpha_{u} \partial_{x} \Phi\left(X_{u}\right) d u & +\int_{s}^{t} \beta_{u} \partial_{x} \Phi\left(X_{u}\right) d B_{u}^{\mathbb{G}} \\
& +\int_{s}^{t}\left(\eta_{u} \partial_{x} \Phi\left(X_{u}\right)+\frac{1}{2} \beta_{u}^{2} \partial_{x x} \Phi\left(X_{u}\right)\right) d\left\langle B^{\mathbb{G}}\right\rangle_{u} .
\end{aligned}
$$

This can be proved by using Taylor series and $\mathbb{G}-$ It $\widehat{o}$ table,

$$
\begin{array}{cccc} 
& \mathrm{dt} & d B_{t}^{\mathbb{G}} & d\left\langle B^{\mathbb{G}}\right\rangle_{t} \\
\mathrm{dt} & 0 & 0 & 0 \\
d B_{t}^{\mathbb{G}} & 0 & d\left\langle B^{\mathbb{G}}\right\rangle_{t} & 0 \\
d\left\langle B^{\mathbb{G}}\right\rangle_{t} & 0 & 0 & 0
\end{array}
$$

2.5. Product formula for $\mathbb{G}-$ Ito processes. For the product $\mathbb{G}$-Itô formula, the technique is same as, it was in case of standard Brownian motion, Let $X_{t}$ and $Y_{t}$ are two $\mathbb{G}$-Itô processes than product $\mathbb{G}$-Itô formula is $[7,2014]$,

$$
d\left(X_{t} Y_{t}\right)=Y_{t} d X_{t}+X_{t} d Y_{t}+d X_{t} d Y_{t}
$$




\section{The WeAther DeRIVATIVES MARKET}

3.1. The Weather Derivative Contract. Weather derivatives are structured as futures, swaps, and put/call options against different underlying weather indices some of them are cooling and heating degreedays (defined in next section), snowfall and rain. But here we will discuss only underlying index temperature(degree days indices). We are giving some definitions and terminology. From now we speak only about the temperature index. Some definitions which we will use in modeling temperature

Definition 3.1. (Temperature) Given a specific weather station, let $T_{i}^{\text {max }}$ and $T_{i}^{\text {min }}$ are the maximal and minimal temperatures(Celsius) of $i^{T h}$ day. We define temperature of day $i$ as

$$
T_{i} \equiv \frac{T_{i}^{\max }+T_{i}^{\min }}{2}
$$

Definition 3.2. (Degree-days) Let $T_{i}$ denote the temperature on day $i$. We define the heating degree-days, $H D D_{i}$ and the cooling degree-days, $C D D_{i}$, as

$$
H D D_{i} \equiv \max \left\{18-T_{i}, 0\right\}
$$

and

$$
C D D_{i} \equiv \max \left\{T_{i}-18,0\right\}
$$

respectively.

In the above definitions, it can be seen that the HDDs and CDDs for a specific day are just the number of degrees that the temperature is deviating from a fixed level called reference level. The names cooling and heating degree-days originate from the US energy sector because if the temperature is below $18^{\circ} \mathrm{C}$ people tend to use more energy to heat their homes, whereas if the temperature is above $18^{\circ} \mathrm{C}$ people start to cool their homes.

Temperature based weather derivatives is based on the accumulation of HDDs or CDDs during a certain period, like one calendar month or a winter/summer period. mostly the HDD season includes the winter months from November to March and CDD season is from May to September. April and October are often referred to as the 'shoulder months'.

3.2. The CME contract. The CME deals with futures based on the CME Degree Day Index, the aggregate amount of a calendar month's average HDDs or CDDs, as well as options for those futures. For more than 11 U.S. cities, the CME Degree Day Index is actually listed.

The futures of the HDD and CDD index are agreements to purchase or sell the HDD and CDD index value at a specific future date. One contract's notional value is $\$ 100$ times the Degree Day Index, and the contracts 
are quoted as HDD and CDD Index points. The futures are cash-settled, meaning that There is index-based regular labeling with the gain or loss added to the customer's account

A CME HDD or CDD call option is a contract that offers the owner the right to buy a HDD / CDD futures contract at a specific price usually called the strike or exercise price, but not the commitment. Analogously, the HDD / CDD put option grants the owner the right to sell one HDD / CDD futures contract, but not the obligation. At the CME, the future options are European style which means that they can only be exercised at the expiration date, Which means they can only be exercised on the expiry date.

3.3. Weather Options. There are several different contracts traded on the OTC market as mentioned above. The option is the common type of contract. Calls and puts are two main types of options.

- A call option is the right to buy a specific asset for an agreed amount at a fixed time in the future, as you must pay the premium at the outset of the deal by buying the right to purchase or not.

- A put option is the right to sell an asset at a fixed time in the future for an agreed amount.

Let someone purchase the call option for some fixed strike level then if the number of HDDs for the contract period is greater than the agreed strike level, the buyer will receive a payout. The size of the payout is determined by the strike and the tick size. The tick size is the amount of money that the holder of the call receives for each degree-day above the strike level for the period. Often the option has a cap on the maximum payout unlike, for example, traditional options on stocks. A generic weather option can be formulated by specifying the following parameters:

- The contract nature (call or put)

- The contract tenure (e.g. December 2019)

- The underlying index (HDD or CDD)

- An official weather station for temperature data

- The strike level

- The tick size

- The maximum payout (if the option is capped)

The aim of this study is to find the formula for the payout of contracted option, let $K$ is the strike level and $\alpha$ is the tick size and contract period is $m$ days. Then the number of CDDs and HDDs for $m$ days period are,

$$
H_{m}=\sum_{i=1}^{m} H D D_{i} \text { and } C_{m}=\sum_{i=1}^{m} C D D_{i} .
$$

Then the formula for payout of uncapped HDD call can be written as

$$
\chi=\alpha \max \left\{H_{m}-K, 0\right\}
$$


Formula for payout of uncapped CDD call can be written as

$$
\chi=\alpha \max \left\{C_{m}-K, 0\right\} .
$$

Payouts of HDD and CDD puts can be defined similarly.

\section{Pricing Weather Derivatives through $\mathbb{G}$-Brownian}

\subsection{Temperature Model under $\mathbb{G}$-framework.}

Theorem 4.1. Let the test model for the temperature under $\mathbb{G}$-Brownian motion and from the Girsanov theorem under $\mathbb{G}$-framwork (cf. [32, 2011]) one can find, the risk neutral measure $\mathbb{Q}$ such that,

$$
d T_{t}=\left(\frac{d T_{t}^{m}}{d t}+a\left(T_{t}^{m}-T_{t}\right)-\eta(t)-\lambda \sigma_{t}\right) d t+\sigma_{t} d B_{t}^{\mathbb{G}}+\eta(t) d\left\langle B^{\mathbb{G}}\right\rangle_{t}
$$

Here $B_{t}^{\mathbb{G}}$ and $\left\langle B^{\mathbb{G}}\right\rangle_{t}$ are $\mathbb{G}$-Brownian motion and quadratic variation of $\mathbb{G}$-Brownian motion respectively, $\eta(t)$ is any integrable deterministic function. Then solution of (4.12),

$$
T_{t}=e^{-a(t-s)}\left(T_{s}+\int_{s}^{t} \beta_{u} e^{-a(s-u)} d u+\int_{s}^{t} \sigma_{u} e^{-a(s-u)} d B_{u}^{\mathbb{G}}+\int_{s}^{t} \eta_{u} e^{-a(s-u)} d\left\langle B^{\mathbb{G}}\right\rangle_{u}\right) .
$$

Moreover, the conditional expectation and conditional variance of $T_{t}$ can be given as,

$$
\widehat{\mathbb{E}}^{\mathbb{G}, \mathbb{Q}}\left[T_{t} \mid \mathcal{F}_{s}\right]=e^{-a(t-s)}\left(T_{s}+\int_{s}^{t} \beta_{u} e^{-a(s-u)} d u+\xi_{\eta}\right) .
$$

where $\xi_{\eta}:=\widehat{\mathbb{E}}^{\mathbb{G}}\left(\int_{s}^{t} \eta_{u} e^{-a(s-u)} d\left\langle B^{\mathbb{G}}\right\rangle_{u} \mid \mathcal{F}_{s}\right)$ and

$$
\begin{aligned}
\operatorname{Var}^{\mathbb{G}, \mathbb{Q}}\left[T_{t} \mid \mathcal{F}_{s}\right] \leq & \bar{\mu}_{T}\left(\bar{\mu}_{T}+2 \widehat{\mu}_{T}\right)+ \\
& e^{-2 a(t-s)}\left(\begin{array}{c}
T_{s}^{2}+I(t)_{\beta}^{2}++\widehat{\mathbb{E}}^{\mathbb{G}, \mathbb{Q}}\left[I\left(\left\langle B^{\mathbb{G}}\right\rangle\right)_{\eta}^{2} \mid \mathcal{F}_{s}\right]+2 T_{s} I(t)_{\beta} \times \\
\int_{s}^{t}\left(2 T_{s} \eta_{u} e^{-a(s-u)}+2 I(t)_{\beta} \eta_{u} e^{-a(s-u)}+\sigma_{u}^{2} e^{-2 a(s-u)}\right) d u
\end{array}\right),
\end{aligned}
$$

where

$$
\begin{aligned}
I(t)_{\beta} & :=\int_{s}^{t} \beta_{u} e^{-a(s-u)} d u, \quad I\left(B^{\mathbb{G}}\right)_{\sigma}=\int_{s}^{t} \sigma_{u} e^{-a(s-u)} d B_{u}^{\mathbb{G}}, \\
I\left(\left\langle B^{\mathbb{G}}\right\rangle\right)_{\eta} & :=\int_{s}^{t} \eta_{u} e^{-a(s-u)} d\left\langle B^{\mathbb{G}}\right\rangle_{u} .
\end{aligned}
$$

Proof. Let us start by rewriting the (4.12) in $\mathbb{G}-$ Ito form,

$$
\begin{aligned}
d T_{t} & =\left(\frac{T_{t}^{m}}{d t}+a\left(T_{t}^{m}-T_{t}\right)-\eta_{t}-\lambda \sigma_{t}\right) d t+\sigma_{t} d B_{t}^{\mathbb{G}}+\eta_{t} d\left\langle B^{\mathbb{G}}\right\rangle_{t}, \\
& =\left(\frac{T_{t}^{m}}{d t}+a T_{t}^{m}-a T_{t}-\eta_{t}-\lambda \sigma_{t}\right) d t+\sigma_{t} d B_{t}^{\mathbb{G}}+\eta_{t} d\left\langle B^{\mathbb{G}}\right\rangle_{t}, \\
& =\left(\left(\frac{T_{t}^{m}}{d t}+a T_{t}^{m}-\eta_{t}-\lambda \sigma_{t}\right)-a T_{t}\right) d t+\sigma_{t} d B_{t}^{\mathbb{G}}+\eta_{t} d\left\langle B^{\mathbb{G}}\right\rangle_{t} .
\end{aligned}
$$


By setting $\beta_{t}=\frac{T_{t}^{m}}{d t}+a T_{t}^{m}-\eta_{t}-\lambda \sigma_{t}$,

$$
d T_{t}=\left[\beta_{t}-a T_{t}\right] d t+\sigma_{t} d B_{t}^{\mathbb{G}}+\eta_{t} d\left\langle B^{\mathbb{G}}\right\rangle_{t},
$$

by solving equation (4.3) through $\mathbb{G}$-Itô formula equation (2.21) and equation (2.22), first solving the homogeneous part of equation (4.3), that is

$$
d T_{t}=-a T_{t} d t
$$

Integrating this from $s$ to $t$,

$$
\begin{aligned}
\ln T_{t}-\ln T_{s} & =-a(t-s) \\
T_{t} & =T_{s} e^{-a(t-s)}
\end{aligned}
$$

Set $\phi_{s, t}=T_{t}-T_{s}$, and solving equation (4.3), by using following transformation.

$$
u\left(t, T_{t}\right)=T_{t} \phi_{s, t}^{-1} .
$$

Applying the product $\mathbb{G}$-Itô formula (i.e. (2.22)) on equation (4.5) and using the $\mathbb{G}$-Itô table 2.4, one can get the following results,

$$
\begin{aligned}
& d\left(T_{t} \phi_{s, t}^{-1}\right)=d T_{t} \phi_{s, t}^{-1}+T_{t} d \phi_{s, t}^{-1}+d T_{t} d \phi_{s, t}^{-1} \\
& d\left(T_{t} \phi_{s, t}^{-1}\right)=\left(\left[\beta_{t}-a T_{t}\right] d t+\sigma_{t} d B_{t}^{\mathbb{G}}+\eta_{t} d\langle B\rangle_{t}\right) \phi_{s, t}^{-1}+a T_{t} \phi_{s, t}^{-1}+0 \\
& d\left(T_{t} \phi_{s, t}^{-1}\right)=\left(\beta_{t} d t+\sigma_{t} d B_{t}^{\mathbb{G}}+\eta_{t} d\langle B\rangle_{t}\right) \phi_{s, t}^{-1} .
\end{aligned}
$$

Integrating equation (4.6) from $s$ to $t$ and by simplification, the solution of equation (4.3) is,

$$
T_{t}=e^{-a(t-s)}\left(T_{s}+\int_{s}^{t} \beta_{u} e^{-a(s-u)} d u+\int_{s}^{t} \sigma_{u} e^{-a(s-u)} d B_{u}^{\mathbb{G}}+\int_{s}^{t} \eta_{u} e^{-a(s-u)} d\left\langle B^{\mathbb{G}}\right\rangle_{u}\right) .
$$

The equation (4.2) represents the stochastic model of temperature in $\mathbb{G}$-framework.

The conditional expected value is,

$$
\begin{aligned}
\widehat{\mathbb{E}}^{\mathbb{G}, \mathbb{Q}}\left[T_{t} \mid \mathcal{F}_{s}\right] & =\widehat{\mathbb{E}}^{\mathbb{G}, \mathbb{Q}}\left[e ^ { - a ( t - s ) } \left(T_{s}+\int_{s}^{t} \beta_{u} e^{-a(s-u)} d u+\int_{s}^{t} \sigma_{u} e^{-a(s-u)} d B_{u}^{\mathbb{G}}\right.\right. \\
& \left.\left.+\int_{s}^{t} \eta_{u} e^{-a(s-u)} d\left\langle B^{\mathbb{G}}\right\rangle_{u}\right) \mid \mathcal{F}_{s}\right] \\
& =e^{-a(t-s)}\left(T_{s}+\int_{s}^{t} \beta_{u} e^{-a(s-u)} d u\right)+\widehat{\mathbb{E}}^{\mathbb{G}, \mathbb{Q}}\left(\int_{s}^{t} e^{-a(s-u)} \sigma_{u} d B_{u}^{\mathbb{G}}\right) \\
& +\widehat{\mathbb{E}}^{\mathbb{G}, \mathbb{Q}}\left(\int_{s}^{t} e^{-a(s-u)} \eta_{u} d\left\langle B^{\mathbb{G}}\right\rangle_{u} \mid \mathcal{F}_{s}\right)
\end{aligned}
$$


Set $\xi_{\eta}:=\widehat{\mathbb{E}}^{\mathbb{G}}\left(\int_{s}^{t} \eta_{u} e^{-a(s-u)} d\left\langle B^{\mathbb{G}}\right\rangle_{u} \mid \mathcal{F}_{s}\right)$ and using properties of sublinear expectations from section 2, Proposition 2.3 and Lemma 2.1,

$$
\widehat{\mathbb{E}}^{\mathbb{G}, \mathbb{Q}}\left[T_{t} \mid \mathcal{F}_{s}\right]=e^{-a(t-s)}\left(T_{s}+\int_{s}^{t} \beta_{u} e^{-a(s-u)} d u+\xi_{\eta}\right) .
$$

The conditional variance can be defined same as it was in linear expectation notion. Let $\bar{\mu}_{T}=\widehat{\mathbb{E}}^{\mathbb{G}}\left[T_{t} \mid \mathcal{F}_{s}\right]$, such that $\bar{\mu}_{T} \geq 0$ also let $\widehat{\mu}_{T}=\widehat{\mathbb{E}}^{\mathbb{G}}\left[-T_{t} \mid \mathcal{F}_{s}\right]$ now variance is

$$
\begin{aligned}
\operatorname{Var}^{\mathbb{G}, \mathbb{Q}}\left[T_{t} \mid \mathcal{F}_{s}\right] & =\widehat{\mathbb{E}}^{\mathbb{G}, \mathbb{Q}}\left[\left(T_{t}-\bar{\mu}_{T}\right)^{2} \mid \mathcal{F}_{s}\right] \\
& =\widehat{\mathbb{E}}^{\mathbb{G}, \mathbb{Q}}\left[T_{t}^{2}+\bar{\mu}_{T}^{2}-2 \bar{\mu}_{T} T_{t} \mid \mathcal{F}_{s}\right] \\
& =\bar{\mu}_{T}^{2}+\widehat{\mathbb{E}}^{\mathbb{G}, \mathbb{Q}}\left[T_{t}^{2}+2 \bar{\mu}_{T}\left[-T_{t}\right] \mid \mathcal{F}_{s}\right] \\
& \leq \bar{\mu}_{T}^{2}+\widehat{\mathbb{E}}^{\mathbb{G}, \mathbb{Q}}\left[T_{t}^{2} \mid \mathcal{F}_{s}\right]+\widehat{\mathbb{E}}^{\mathbb{G}, \mathbb{Q}}\left[2 \bar{\mu}_{T}\left(-T_{t}\right) \mid \mathcal{F}_{s}\right] \\
& =\bar{\mu}_{T}^{2}+\widehat{\mathbb{E}}^{\mathbb{G}, \mathbb{Q}}\left[T_{t}^{2} \mid \mathcal{F}_{s}\right]+2 \bar{\mu}_{T} \widehat{\mathbb{E}}^{\mathbb{G}, \mathbb{Q}}\left[-T_{t} \mid \mathcal{F}_{s}\right] \\
& =\bar{\mu}_{T}^{2}+\widehat{\mathbb{E}}^{\mathbb{G}, \mathbb{Q}}\left[T_{t}^{2} \mid \mathcal{F}_{s}\right]+2 \bar{\mu}_{T} \widehat{\mu}_{T} \\
\operatorname{Var}^{\mathbb{G}, \mathbb{Q}}\left[T_{t} \mid \mathcal{F}_{s}\right] & \leq \bar{\mu}_{T}\left(\bar{\mu}_{T}+2 \widehat{\mu}_{T}\right)+\widehat{\mathbb{E}}^{\mathbb{G}, \mathbb{Q}}\left[T_{t}^{2} \mid \mathcal{F}_{s}\right] .
\end{aligned}
$$

Let $N=\bar{\mu}_{T}\left(\bar{\mu}_{T}+2 \widehat{\mu}_{T}\right)+\widehat{\mathbb{E}}^{\mathbb{G}}\left[T_{t}^{2} \mid \mathcal{F}_{s}\right]$ and from the fact that $\operatorname{Var}\left[T_{t} \mid \mathcal{F}_{s}\right] \geq 0$ one can write it as $\bar{\Sigma}^{2}=$ $\operatorname{Var}\left[T_{t} \mid \mathcal{F}_{s}\right] \in[0, N]$. Here $\widehat{\mathbb{E}}^{\mathbb{G}, \mathbb{Q}}\left[T_{t}^{2} \mid \mathcal{F}_{s}\right]$ can be estimated by following approximation of it, but for the sake of simplicity there are some conventional notations have been defined as, let

$$
\begin{aligned}
I(t)_{\beta} & :=\int_{s}^{t} \beta_{u} e^{-a(s-u)} d u, I\left(B^{\mathbb{G}}\right)_{\sigma}:=\int_{s}^{t} \sigma_{u} e^{-a(s-u)} d B_{u}^{\mathbb{G}}, \\
I\left(\left\langle B^{\mathbb{G}}\right\rangle\right)_{\eta} & :=\int_{s}^{t} \eta_{u} e^{-a(s-u)} d\left\langle B^{\mathbb{G}}\right\rangle_{u} .
\end{aligned}
$$

Now

$$
\begin{aligned}
& \widehat{\mathbb{E}}^{\mathbb{G}, \mathbb{Q}}\left[T_{t}^{2} \mid \mathcal{F}_{s}\right]=\widehat{\mathbb{E}}^{\mathbb{G}, \mathbb{Q}}\left[\left(e ^ { - a ( t - s ) } \left(T_{s}+\int_{s}^{t} \beta_{u} e^{-a(s-u)} d u+\int_{s}^{t} \sigma_{u} e^{-a(s-u)} d B_{u}^{\mathbb{G}}\right.\right.\right. \\
& \left.\left.\left.+\int_{s}^{t} \eta_{u} e^{-a(s-u)} d\left\langle B^{\mathbb{G}}\right\rangle_{u}\right)\right)^{2} \mid \mathcal{F}_{s}\right] \\
& =e^{-2 a(t-s)} \widehat{\mathbb{E}}^{\mathbb{G}, \mathbb{Q}}\left[T_{s}^{2}+I(t)_{\beta}^{2}+I\left(B^{\mathbb{G}}\right)_{\sigma}^{2}+I\left(\left\langle B^{\mathbb{G}}\right\rangle\right)_{\eta}^{2}+2 T_{s} I(t)_{\beta}+2 T_{s} I\left(B^{\mathbb{G}}\right)_{\sigma}\right. \\
& \left.+2 T_{s} I\left(\left\langle B^{\mathbb{G}}\right\rangle\right)_{\eta}+2 I(t)_{\beta} I\left(B^{\mathbb{G}}\right)_{\sigma}+2 I(t)_{\beta} I\left(\left\langle B^{\mathbb{G}}\right\rangle\right)_{\eta}+2 I\left(B^{\mathbb{G}}\right)_{\sigma} I\left(\left\langle B^{\mathbb{G}}\right\rangle\right)_{\eta} \mid \mathcal{F}_{s}\right],
\end{aligned}
$$

using properties of sublinear expectations from section 2, Proposition 2.3 and Lemma 2.1,

$$
\begin{aligned}
\widehat{\mathbb{E}}^{\mathbb{G}, \mathbb{Q}}\left[T_{t}^{2} \mid \mathcal{F}_{s}\right] & \leq e^{-2 a(t-s)}\left(T_{s}^{2}+I(t)_{\beta}^{2}+2 T_{s} I(t)_{\beta}+2 T_{s} \widehat{\mathbb{E}}^{\mathbb{G}, \mathbb{Q}}\left[I\left(B^{\mathbb{G}}\right)_{\sigma} \mid \mathcal{F}_{s}\right]\right. \\
& +2 T_{s} \widehat{\mathbb{E}}^{\mathbb{G}, \mathbb{Q}}\left[I\left(\left\langle B^{\mathbb{G}}\right\rangle\right)_{\eta} \mid \mathcal{F}_{s}\right]+2 I(t)_{\beta} \widehat{\mathbb{E}}^{\mathbb{G}, \mathbb{Q}}\left[I\left(B^{\mathbb{G}}\right)_{\sigma} \mid \mathcal{F}_{s}\right]+2 I(t)_{\beta} \widehat{\mathbb{E}}^{\mathbb{G}, \mathbb{Q}}\left[I\left(\left\langle B^{\mathbb{G}}\right\rangle\right)_{\eta} \mid \mathcal{F}_{s}\right] \\
& \left.\left.+\widehat{\mathbb{E}}^{\mathbb{G}, \mathbb{Q}}\left[I\left(B^{\mathbb{G}}\right)_{\sigma}^{2} \mid \mathcal{F}_{s}\right]+\widehat{\mathbb{E}}^{\mathbb{G}, \mathbb{Q}} I\left(\left\langle B^{\mathbb{G}}\right\rangle\right)_{\eta}^{2} \mid \mathcal{F}_{s}\right]+2 \widehat{\mathbb{E}}^{\mathbb{G}, \mathbb{Q}}\left[I\left(B^{\mathbb{G}}\right)_{\sigma} I(\langle B\rangle)_{\eta} \mid \mathcal{F}_{s}\right]\right)
\end{aligned}
$$


Using some properties from [24, 2007], and properties of sublinear expectations from section 2, Proposition 2.3 and Lemma 2.1,

$$
\begin{aligned}
& \widehat{\mathbb{E}}^{\mathbb{G}, \mathbb{Q}}\left[T_{t}^{2} \mid \mathcal{F}_{s}\right] \leq e^{-2 a(t-s)}\left(\begin{array}{c}
T_{s}^{2}+I(t)_{\beta}^{2}+2 T_{s} I(t)_{\beta}+2 T_{s} \int_{s}^{t} \eta_{u} e^{-a(s-u)} d u \\
+2 I(t)_{\beta} \int_{s}^{t}\left|\eta_{u}\right| e^{-a(s-u)} d u+\int_{s}^{t} \sigma_{u}^{2} e^{-2 a(s-u)} d u \\
+\widehat{\mathbb{E}}^{\mathbb{G}, \mathbb{Q}}\left[I\left(\left\langle B^{\mathbb{G}}\right\rangle\right)_{\eta}^{2} \mid \mathcal{F}_{s}\right]
\end{array}\right), \\
& \leq e^{-2 a(t-s)}\left(\begin{array}{l}
T_{s}^{2}+I(t)_{\beta}^{2}+2 T_{s} I(t)_{\beta}+2 T_{s} \int_{s}^{t} \eta_{u} e^{-a(s-u)} d u \\
+2 I(t)_{\beta} \int_{s}^{t} \eta_{u} e^{-a(s-u)} d u+\int_{s}^{t} \sigma_{u}^{2} e^{-2 a(s-u)} d u+ \\
\widehat{\mathbb{E}}^{\mathbb{G}, \mathbb{Q}}\left[I\left(\left\langle B^{\mathbb{G}}\right\rangle\right)_{\eta}^{2} \mid \mathcal{F}_{s}\right]
\end{array}\right) \\
& \leq e^{-2 a(t-s)}\left(\begin{array}{l}
T_{s}^{2}+I(t)_{\beta}^{2}+2 T_{s} I(t)_{\beta}+2 T_{s} \int_{s}^{t} \eta_{u} e^{-a(s-u)} d u \\
+2 I(t)_{\beta} \int_{s}^{t} \eta_{u} e^{-a(s-u)} d u+\int_{s}^{t} \sigma_{u}^{2} e^{-2 a(s-u)} d u+ \\
\widehat{\mathbb{E}}^{\mathbb{G}, \mathbb{Q}}\left[I\left(\left\langle B^{\mathbb{G}}\right\rangle\right)_{\eta}^{2} \mid \mathcal{F}_{s}\right]
\end{array}\right) . \\
& \leq e^{-2 a(t-s)}\left(\begin{array}{l}
T_{s}^{2}+I(t)_{\beta}^{2}+2 T_{s} I(t)_{\beta} \times \\
\int_{s}^{t}\left(2 T_{s} \eta_{u} e^{-a(s-u)}+2 I(t)_{\beta} \eta_{u} e^{-a(s-u)}+\sigma_{u}^{2} e^{-2 a(s-u)}\right) d u \\
+\widehat{\mathbb{E}}^{\mathbb{G}, \mathbb{Q}}\left[I\left(\left\langle B^{\mathbb{G}}\right\rangle\right)_{\eta}^{2} \mid \mathcal{F}_{s}\right]
\end{array}\right) .
\end{aligned}
$$

Substituting the value of

$$
\begin{aligned}
\operatorname{Var}^{\mathbb{G}, \mathbb{Q}}\left[T_{t} \mid \mathcal{F}_{s}\right] \leq & \bar{\mu}_{T}\left(\bar{\mu}_{T}+2 \widehat{\mu}_{T}\right)+e^{-2 a(t-s)} \times \\
& \left(\begin{array}{c}
T_{s}^{2}+I(t)_{\beta}^{2}+2 T_{s} I(t)_{\beta} \times \\
\int_{s}^{t}\left(2 T_{s} \eta_{u} e^{-a(s-u)}+2 I(t)_{\beta} \eta_{u} e^{-a(s-u)}+\sigma_{u}^{2} e^{-2 a(s-u)}\right) d u \\
+\widehat{\mathbb{E}}^{\mathbb{G}, \mathbb{Q}}\left[I\left(\left\langle B^{\mathbb{G}}\right\rangle\right)_{\eta}^{2} \mid \mathcal{F}_{s}\right]
\end{array}\right) .
\end{aligned}
$$

\subsection{Pricing Weather Derivative.}

Theorem 4.2. Suppose that dynamics of temperatures process $\left(T_{t}\right)_{t \geq 0}$ satisfies following the $\mathbb{G}$-SDE,

$$
d T_{t}=\left(\frac{d T_{t}^{m}}{d t}+a\left(T_{t}^{m}-T_{t}\right)-\eta(t)-\lambda \sigma_{t}\right) d t+\sigma_{t} d B_{t}^{\mathbb{G}}+\eta(t) d\left\langle B^{\mathbb{G}}\right\rangle_{t}
$$

Then the risk-neutral (arbitrage free) price $H C_{t}$ of the uncapped HDD call (described in section 3) satisfies the following estimate,

$$
H C_{t} \leq 18 m \alpha-e^{-\left(t_{m}-t\right)} K \alpha-\sum_{i=1}^{m} \alpha\left(e^{-a\left(t_{i}-s\right)}\left(T_{t_{i}}+\int_{s}^{t_{i}} \beta_{u} e^{-a\left(t_{i}-u\right)} d u+\xi_{\eta}\left(t_{i}\right)\right)\right) .
$$


and the risk-neutral (arbitrage free) price $C C_{t}$ of the uncapped $C D D$ call (described in section 3) satisfies the following estimate,

$$
C C_{t} \leq e^{-\left(t_{m}-t\right)} K \alpha+18 m \alpha+\sum_{i=1}^{m}\left(\alpha e^{-a\left(t_{i}-s\right)}\left(T_{t_{i}}+\int_{s}^{t_{i}} \beta_{u} e^{-a\left(t_{i}-u\right)} d u \xi_{\eta}\left(t_{i}\right)\right)\right) .
$$

where $\xi_{\eta}=\widehat{\mathbb{E}}^{\mathbb{G}, \mathbb{Q}}\left(\int_{s}^{t} \eta_{u} e^{-a(s-u)} d\left\langle B^{\mathbb{G}}\right\rangle_{u} \mid \mathcal{F}_{s}\right)$.

Proof. Let us recall, from section 3, the payout of HDD call with tick size $\alpha$ is

$$
\chi=\alpha\left(H_{m}-K\right)^{+}
$$

where $H_{m}=\sum_{i=1}^{m} \max \left(18-T_{t_{i}}\right)$, it is known that $T_{t}$ is $\mathbb{G}$-Normally distributed but the $H_{m}$ is not $\mathbb{G}$ Normally distributed just because there is a maximum in its definition, so just for the simplicity if someone is interested to find out the explicit formula for option, then it will be possible for winter months and with an assumption that $18-T_{t_{i}} \geq 0$. Now just to be precise, let for winter months $H_{m}=18 m-\sum_{i=1}^{m} T_{t_{i}}$, which is $\mathbb{G}$-normally distributed.

$$
\begin{aligned}
\widehat{\mathbb{E}}^{\mathbb{G}, \mathbb{Q}}\left[H_{m} \mid \mathcal{F}_{t}\right] & =\widehat{\mathbb{E}}^{\mathbb{G}, \mathbb{Q}}\left[18 m-\sum_{i=1}^{m} T_{t_{i}} \mid \mathcal{F}_{t}\right]=\widehat{\mathbb{E}}^{\mathbb{G}, \mathbb{Q}}[18 m]+\widehat{\mathbb{E}}^{\mathbb{G}, \mathbb{Q}}\left[\sum_{i=1}^{m}\left(-T_{t_{i}}\right) \mid \mathcal{F}_{t}\right] \\
& \leq 18 m+\sum_{i=1}^{m} \widehat{\mathbb{E}}^{\mathbb{G}, \mathbb{Q}}\left[-T_{t_{i}} \mid \mathcal{F}_{t}\right]
\end{aligned}
$$

Set

$$
\begin{aligned}
\bar{\mu}_{m}=\widehat{\mathbb{E}}^{\mathbb{G}, \mathbb{Q}}\left[H_{m} \mid \mathcal{F}_{t}\right], & \bar{\mu}_{m}=\widehat{\mathbb{E}}^{\mathbb{G}, \mathbb{Q}}\left[-H_{m} \mid \mathcal{F}_{t}\right], \\
\widehat{\sigma}_{m}^{2}=\operatorname{Var}\left[H_{m} \mid \mathcal{F}_{t}\right], & \widehat{\sigma}_{m}^{2}=-\operatorname{Var}\left[-H_{m} \mid \mathcal{F}_{t}\right] .
\end{aligned}
$$

Now from Feynman-Kac Formula (cf. [27])

$$
H C_{t}=\widehat{\mathbb{E}}^{\mathbb{G}}\left(\alpha e^{-\left(t_{m}-t\right)}\left(H_{m}-K\right)^{+} \mid \mathcal{F}_{t}\right)
$$

The case when the derivative is in the money i.e. temperature is above 18 for some days, then

$$
\begin{aligned}
H C_{t} & =\alpha e^{-\left(t_{m}-t\right)} \widehat{\mathbb{E}}^{\mathbb{G}, \mathbb{Q}}\left(\left(H_{m}-K\right) \mid \mathcal{F}_{t}\right)=\alpha e^{-\left(t_{m}-t\right)} \widehat{\mathbb{E}}^{\mathbb{G}, \mathbb{Q}}\left(H_{m} \mid \mathcal{F}_{t}\right)-e^{-\left(t_{m}-t\right)} K, \\
& \leq 18 m \alpha-e^{-\left(t_{m}-t\right)} K \alpha+\sum_{i=1}^{m} \alpha \widehat{\mathbb{E}}^{\mathbb{G}, \mathbb{Q}}\left[-T_{t_{i}} \mid \mathcal{F}_{t}\right] \\
& \leq 18 m \alpha-e^{-\left(t_{m}-t\right)} K \alpha-\sum_{i=1}^{m}\left(\alpha e^{-a\left(t_{i}-s\right)}\left(T_{t_{i}}+\int_{s}^{t_{i}} \beta_{u} e^{-a\left(t_{i}-u\right)} d u+\xi_{\eta}\left(t_{i}\right)\right)\right) .
\end{aligned}
$$

Now lets move towards, uncapped CDD call. Recall the payoff of the uncapped CCD call,

$$
\chi=\alpha\left(K-C_{m}\right)^{+},
$$


where $C_{m}=\sum_{i=1}^{m} \max \left(T_{t_{i}-18}\right)$, it is known that $T_{t}$ is $\mathbb{G}$-Normally distributed but the $H_{m}$ is not $\mathbb{G}$-Normally distributed, so to find out the explicit formula for option, then it will be possible for summer months there can be days where the temperature goes above 18 i.e. $T_{t_{i}}-18 \geq 0$. Now just to be precise, let for winter months $C_{m}=\sum_{i=1}^{m} T_{t_{i}}-18 m$, which is $\mathbb{G}$-normally distributed.

$$
\begin{aligned}
\widehat{\mathbb{E}}^{\mathbb{G}, \mathbb{Q}}\left[C_{m} \mid \mathcal{F}_{t}\right] & =\widehat{\mathbb{E}}^{\mathbb{G}, \mathbb{Q}}\left[\sum_{i=1}^{m} T_{t_{i}}-18 m \mid \mathcal{F}_{t}\right]=\widehat{\mathbb{E}}^{\mathbb{G}, \mathbb{Q}}\left[\sum_{i=1}^{m}\left(T_{t_{i}}\right) \mid \mathcal{F}_{t}\right]-18 m \\
& \leq \sum_{i=1}^{m} \widehat{\mathbb{E}}^{\mathbb{G}, \mathbb{Q}}\left[T_{t_{i}} \mid \mathcal{F}_{t}\right]-18 m
\end{aligned}
$$

Now from Feynman-Kac Formula (cf. [27])

$$
C_{t}=\widehat{\mathbb{E}}^{\mathbb{G}, \mathbb{Q}}\left(\alpha e^{-\left(t_{m}-t\right)}\left(K-C_{m}\right)^{+} \mid \mathcal{F}_{t}\right)
$$

The case when the derivative is in the money i.e. temperature is above 18 for some days, then

$$
\begin{aligned}
C C_{t} & =\alpha e^{-\left(t_{m}-t\right)} \widehat{\mathbb{E}}^{\mathbb{G}}\left(\left(K-C_{m}\right) \mid \mathcal{F}_{t}\right) \\
& =e^{-\left(t_{m}-t\right)} K \alpha-\alpha e^{-\left(t_{m}-t\right)} \widehat{\mathbb{E}}^{\mathbb{G}, \mathbb{Q}}\left(C_{m} \mid \mathcal{F}_{t}\right), \\
& \leq e^{-\left(t_{m}-t\right)} K \alpha+18 m \alpha+\sum_{i=1}^{m} \alpha \widehat{\mathbb{E}}^{\mathbb{G}, \mathbb{Q}}\left[T_{t_{i}} \mid \mathcal{F}_{t}\right] \\
& \leq e^{-\left(t_{m}-t\right)} K \alpha+18 m \alpha+\sum_{i=1}^{m}\left(\alpha e^{-a\left(t_{i}-s\right)}\left(T_{t_{i}}+\int_{s}^{t_{i}} \beta_{u} e^{-a\left(t_{i}-u\right)} d u+\xi_{\eta}\left(t_{i}\right)\right)\right) .
\end{aligned}
$$

Conflicts of Interest: The author(s) declare that there are no conflicts of interest regarding the publication of this paper.

\section{REFERENCES}

[1] P. Alaton, B. Djehiche, D. Stillberger, On modelling and pricing weather derivatives, Appl. Math. Finance. 9 (2002), 1-20.

[2] P. Artzner, F. Delbaen, J.-M. Eber, D. Heath, Coherent Measures of Risk, Math. Finance. 9 (1999), 203-228.

[3] M. Avellaneda, A. Levy, A. ParÁS, Pricing and hedging derivative securities in markets with uncertain volatilities, Appl. Math. Finance. 2 (1995), 73-88.

[4] A. Alexandridis, A.D. Zapranis, Weather derivatives: modeling and pricing weather-related risk. Springer Science \& Business Media, New York, (2012).

[5] T.G. Bali, S.J. Brown, Y. Tang, Is economic uncertainty priced in the cross-section of stock returns?, J. Financ. Econ. 126 (2017), 471-489.

[6] Z. Chen, L. Epstein, Ambiguity, Risk, and Asset Returns in Continuous Time, Econometrica. 70 (2002), $1403-1443$.

[7] P. Glasserman, X. Xu, Robust risk measurement and model risk, Quant. Finance. 14 (2014), 29-58.

[8] M.A. Soomro, J. Hussain, On Study of Generalized Novikov Equation by Reduced Differential Transform Method, Indian J. Sci. Technol. 12 (2019), 1-6. 
[9] J. Hussain, B. Khan, On Cox-Ross-Rubinstein Pricing Formula for Pricing Compound Option, Int. J. Anal. Appl. 18 (1) (2020), 129-148.

[10] J. Hussain, M.S. Khan, On the Pricing of Call-Put Parities of Asian Options by Reduced Differential Transform Algorithm, Int. J. Anal. Appl. 18 (3) (2020), 513-530.

[11] J. Hussain, Valuation of European Style Compound Option Written on European Style Currency and Power Options, Int. J. Anal. Appl. 18 (6) (2020), 1015-1028.

[12] J. Hussain, On Existence and invariance of sphere, of solutions of constrained evolution equation, Int. J. Math. Comput. Sci. 15 (2020), 325-345.

[13] M.-U. Rehman, J. Alzabut, J.H. Brohi, A. Hyder, On Spectral Properties of Doubly Stochastic Matrices, Symmetry. 12 (2020), 369.

[14] M.-U. Rehman, J. Alzabut, J. Hussain Brohi, Computing $\mu$-values for LTI Systems, AIMS Math. 6 (2021), $304-313$.

[15] J. Hölzermann, Pricing Interest Rate Derivatives under Volatility Uncertainty, ArXiv:2003.04606 [q-Fin]. (2020).

[16] K. Ito, Differential equations determining a markoff process. Kiyosi Itô Selected Papers (DW Stroock and SRS Varadhan, eds.), Springer-Verlag, pp. 42-75. 1987.

[17] L. Jiang, Convexity, translation invariance and subadditivity for $G$-expectations and related risk measures, Ann. Appl. Probab. 18 (2008), 245-258.

[18] G. Leobacher, P. Ngare, On Modelling and Pricing Rainfall Derivatives with Seasonality, Appl. Math. Finance. 18 (2011), $71-91$.

[19] T.J. Lyons, Uncertain volatility and the risk-free synthesis of derivatives, Appl. Math. Finance. 2 (1995), $117-133$.

[20] S. Marginson M. Considine. The Enterprise University: Power, Governance and Reinvention in Australia Cambridge University Press Cambridge. (2000).

[21] J. Möllmann, M. Buchholz, O. Musshoff, Comparing the hedging effectiveness of weather derivatives based on remotely sensed vegetation health indices and meteorological indices. Weather Climate Soc. 11 (2019), 33-48.

[22] F. Pérez-González, H. Yun, Risk Management and Firm Value: Evidence from Weather Derivatives: Risk Management and Firm Value, J. Finance. 68 (2013), 2143-2176.

[23] M. Ritter, O. Mußhoff, M. Odening, Minimizing Geographical Basis Risk of Weather Derivatives Using A Multi-Site Rainfall Model, Comput. Econ. 44 (2014), 67-86.

[24] S. Peng, G-Brownian Motion and Dynamic Risk Measure under Volatility Uncertainty, ArXiv:0711.2834 [Math]. (2007).

[25] S. Peng, Multi-dimensional G-Brownian motion and related stochastic calculus under G-expectation, Stoch. Proc. Appl. 118 (2008), 2223-2253.

[26] S. Peng, Backward stochastic differential equation, nonlinear expectation and their applications, Proceedings of the International Congress of Mathematicians 2010, pp. 393-432, (2010).

[27] S. Peng, Nonlinear Expectations and Stochastic Calculus under Uncertainty: with Robust CLT and G-Brownian Motion, Springer Berlin Heidelberg, 2019.

[28] A. Salgueiro, M. T. Rodon, Approaching rainfall-based weather derivatives pricing and operational challenges, Rev. Deriv. Res. 23 (2020), 163-190.

[29] I. Štulec, Effectiveness of Weather Derivatives as a Risk Management Tool in Food Retail: The Case of Croatia, Int. J. Financ. Stud. 5 (2017), 2.

[30] K. E. Trenberth. The definition of el nino. Amer. Meteorol. Soc. 78 (12) (1997), 2771-2778.

[31] J. Xu, M.P. Xu, European Call Option Price under G-Framework. Math. Practice Theory, 4 (2010), 41-45.

[32] J. Xu, H. Shang, B. Zhang, A Girsanov Type Theorem Under G-Framework, Stoch. Anal. Appl. 29 (2011), 386-406. 
[33] J. Yang, W. Zhao, Numerical simulations for G-Brownian motion, Front. Math. China. 11 (2016), 1625-1643. 